\title{
Combings of groups and the grammar of reparameterization
}

\author{
Martin R. Bridson
}

\begin{abstract}
A new construction of combings is used to distinguish between several previously indistinguishable classes of groups associated to the theory of automatic groups and non-positive curvature in group theory. We construct synchronously bounded combings for a class of groups that are neither bicombable nor automatic. The linguistic complexity of these combings is analysed: in many cases the language of words in the combing is an indexed language.
\end{abstract}

Mathematics Subject Classification (2000). 20F10, 20F65, 20F67, 68Q42, 68Q45, 03D40.

Keywords. Finitely presented groups, combings, automatic groups, indexed languages.

\section{Introduction}

Geometric group theory has enjoyed a period of rapid and exciting growth in the decade or so since the emergence of the theory of hyperbolic groups [20] and the theory of automatic groups [15]. However, the subject has been marred by the absence of examples to distinguish between the various classes of groups defined in terms of the geometry and linguistic complexity of the normal forms (combings) which they admit (cf. page 83 of [15] and section 6 of [21]). In particular, there have been no examples to distinguish the class of combable groups from that of bicombable groups, likewise combable groups versus automatic groups, automatic groups versus biautomatic groups, and $\mathcal{A}$-combable groups versus automatic groups, where $\mathcal{A}$ is any full abstract family of languages.

In this article we introduce a new method for constructing combings (see Theorem 3.4). We shall use the combings constructed by this method to distinguish between several previously indistinguishable classes of groups, thus responding to questions raised by Epstein et al. ([15] p. 85), Gersten et al. [16], and others (e.g. [29]). In particular we shall prove:

Theorem A. There exist combable groups that are not bicombable.

The author's research is supported by an EPSRC Advanced Fellowship. 
Theorem B. There exist combable groups whose Dehn functions are cubic.

Corollary C. There exist combable groups that are not automatic.

I should emphasize that although I am using the definition of combability that has now become standard, it is not the definition first used by Epstein and Thurston, which leads to a quadratic isoperimetric inequality (see pages 83-86 of [15]). Thus, given a group $\Gamma$ with finite generating set $\Sigma$, we say that $\Gamma$ is combable if one can make a choice of words $(\sigma(\gamma): \gamma \in \Gamma)$ in the letters $\Sigma^{ \pm 1}$ such that $\sigma(\gamma)=\gamma$ in $\Gamma$ and the fellow-traveller property holds: when viewed as an edge-path from 1 to $\gamma$ in the Cayley graph of $\Gamma, \sigma(\gamma)$ remains uniformly close to $\sigma\left(\gamma^{\prime}\right)$ if the vertex $\gamma^{\prime}$ is adjacent to $\gamma$.

In Section 6 we shall analyse the linguistic complexity of the combings constructed in the proof of the above theorems. In many cases the language of words in the combing is shown to be an indexed language. Recall that the full abstract families consisting of regular languages, context-free languages and indexed languages form a hierarchy Reg $\subset \mathrm{CF} \subset$ Ind (see Section 5). Regular languages form the basis of automatic group theory (see [15]), and the work of Bridson and Gilman [10] shows that within the class of indexed languages one can construct efficient combings for the fundamental groups of arbitrary compact, geometrizable 3-manifolds.

Theorem D. There exist Ind-combable groups that are not Reg-combable (i.e. automatic).

Here is an explicit example of a group that is Ind-combable but neither bicombable nor automatic. (Large classes of examples will be described in Sections 4 and 6 - see in particular 4.2 and 6.4.) This group has a cubic Dehn function and is the fundamental group of a compact aspherical 2-complex. It is obtained by taking two direct products of free groups $F\left(a_{i}, b_{i}\right) \times F\left(s_{i}, t_{i}\right)$ and forming their amalgamated free product along the subgroups generated by $\left\{s_{i}, t_{i} a_{i}\right\}$ :

$$
\left\langle a_{1}, b_{1}, s, t_{1}, a_{2}, b_{2}, t_{2} \mid t_{1} a_{1}=t_{2} a_{2},\left[a_{i}, s\right]=\left[a_{i}, t_{i}\right]=\left[b_{i}, s\right]=\left[b_{i}, t_{i}\right]=1, i=1,2\right\rangle .
$$

One shows that this group is not bicombable by proving that the centralizer of $\left\{a_{1}, a_{2}, b_{1}, b_{2}\right\}$ is not finitely generated.

Bestvina and Brady have outlined a different construction of a combable group that is not automatic. Their example has a quadratic Dehn function.

In the course of our project on subgroups of automatic groups, [3] and [4], Baumslag, Miller, Short and I shared many stimulating conversations about combings of groups, and I thank them for the seeds of thought that undoubtedly found their way through those conversations into this article. I thank Bob Gilman for the insights into the utility of formal grammars that I gained from him in the course of our joint project [10] and [9]. I thank both Lawrence Reeves and the referee for their careful reading and useful comments. 


\section{Combings}

A choice of generating set for a group $G$ is a surjective monoid homomorphism $\mu: \Sigma^{*} \rightarrow G$, where $\Sigma^{*}$ is the free monoid on the set $\Sigma$. In the following discussion we assume that $\Sigma$ is finite.

It is convenient to assume that $\Sigma$ is equipped with an involution, written $s \mapsto$ $s^{-1}$, such that $\mu\left(s^{-1}\right)=\mu(s)^{-1}$, and we shall do so without further comment. We write $|w|$ to denote the length of a word $w \in \Sigma^{*}$.

A combing of $G$ is a map $\sigma: G \rightarrow \Sigma^{*}$ such that $\mu \circ \sigma(g)=g$ for all $g \in G$. It is often fruitful to regard $\sigma(g)$ as a choice of discrete path from $1 \in G$ to $g$ : at integer times $t \leq|\sigma(g)|$ this path visits the group element $\mu\left(\sigma(g)_{t}\right)$, where $w_{t}$ denotes the prefix of length $t$ in a word $w$.

The combing is said to satisfy the (synchronous) fellow-traveller property if the paths to adjacent vertices stay uniformly close together. More precisely, there should exist a constant $K>0$ such that for all $g, g^{\prime} \in G$,

$$
d\left(\mu\left(\sigma(g)_{t}\right), \mu\left(\sigma\left(g^{\prime}\right)_{t}\right)\right) \leq K d\left(g, g^{\prime}\right)
$$

for all $t \leq \max \left\{|\sigma(g)|,\left|\sigma\left(g^{\prime}\right)\right|\right\}$, where $d$ is the word metric associated to our choice of generators, i.e. the unique left-invariant metric on $G$ such that $d(1, g)$ is the length of the shortest word in $\mu^{-1}(g)$. If a finitely generated group $G$ admits such a combing, it is said to be combable. It is called bicombable if it satisfies the following strengthening of the above inequality:

$$
d\left(\mu\left(s . \sigma\left(s^{-1} g s^{\prime}\right)_{t}\right), \mu\left(\sigma(g)_{t}\right)\right) \leq K
$$

for all $s, s^{\prime} \in \Sigma$ and $g \in G$; and in this case $\sigma$ is called a bicombing. A bicombing is said to have the comparable lengths property if there is a constant $C$ such that translated combing paths that begin and end a distance at most one apart have lengths that differ by at most $C$, that is

$$
|| \sigma\left(s^{-1} g s^{\prime}\right)|-| \sigma(g)|| \leq C
$$

for all $g \in G$ and $s, s^{\prime} \in \Sigma \cup\{1\}$. A combing is said to have the comparable lengths property if (1.3) holds with $s \equiv 1$.

Let $\mathcal{A}$ be a full AFL (see 5.1). One says that $\sigma$ is an $\mathcal{A}$-combing if it satisfies the fellow-traveller property and the image of $\sigma$ is a language in the class $\mathcal{A}$; if such a $\sigma$ exists, one says that $G$ is $\mathcal{A}$-combable. $\mathcal{A}$-bicombings and $\mathcal{A}$-bicombable groups are defined similarly.

In the special case $\mathcal{A}=\operatorname{Reg}$, an $\mathcal{A}$-combing is called an automatic structure for $G$, and an $\mathcal{A}$-bicombing is called a biautomatic structure for $G$ (see [15]).

Remark 1.1. (1) It is easy to check that all of the above properties are independent of the chosen finite generating set for the group. In other words, given a finite generating set for a group, if the group admits a certain type of combing with respect to that set of generators, then it admits such a combing with respect to any other finite set of generators. 
(2) Although it is not the focus of this article, for completeness I should mention that there are many situations in which it is natural to allow reparameterizations of paths in the definition of the fellow-traveller property. This relaxation of the fellow-traveller condition results in the (weaker) notion of an asynchronously $\mathcal{A}$ combable group — see [15], [10] and 6.5(3).

\subsection{Basic properties}

We remind the reader that the Dehn function $\delta(n)$ of a finitely presented group $\Gamma=\langle\Sigma \mid \mathcal{R}\rangle$ measures the complexity of the word problem in $\Gamma$. More precisely, it gives the optimal bound on the number of relators $r \in \mathcal{R}$ that one must apply in order to show that a word in the free group $F(\Sigma)$ is null-homotopic, i.e. represents $1 \in \Gamma$. Thus if $N$ is the least integer such that for every null-homotopic word $w$ of length $\leq n$ there is an equality $w=\prod_{i=1}^{M} x_{i}^{-1} r_{i} x_{i}$ in $F(\Sigma)$, with $r_{i} \in \mathcal{R}^{ \pm 1}$ and $M \leq N$, then $\delta(n)=N$. Up to a standard equivalence relation, the Dehn function of a group is independent of the chosen finite presentation.

The following standard result is proved using diagrammatic techniques (see, for example, [15] or [6]).

Lemma 1.2. If a finitely generated group $G$ admits a combing $\sigma$ that satisfies the fellow-traveller property then:

(1) $G$ is finitely presented;

(2) $G$ has a solvable word problem and its Dehn function is bounded above by a constant times $n L_{\sigma}(n)$, where $L_{\sigma}(n)$ is the length function of $\sigma$ :

$$
L_{\sigma}(n):=\max \{|\sigma(g)| \mid d(1, g) \leq n\} .
$$

(Note that if $\sigma$ has the comparable lengths property then $L_{\sigma}(n) \simeq n$.)

We shall also need the following standard facts.

\section{Lemma 1.3.}

(1) Every (bi)automatic structure has the comparable lengths property.

(2) If $G_{1}$ and $G_{2}$ are biautomatic, then so is $G_{1} \times G_{2}$. More generally, given any full abstract family of languages $\mathcal{A}$, if $G_{1}$ and $G_{2}$ are $\mathcal{A}$-bicombable, then so is $G_{1} \times G_{2}$.

Proof. The first assertion is an easy consequence of the interpretation of regular languages as precisely the languages accepted by finite state automata - see [15]. Given bicombings $\sigma^{j}: G_{j} \rightarrow \Sigma_{j}^{*}$, one obtains a bicombing of $G_{1} \times G_{2}$ by defining $\sigma\left(g_{1}, g_{2}\right)=\sigma^{1}\left(g_{1}\right) \sigma^{2}\left(g_{2}\right) \in\left(\Sigma_{1} \amalg \Sigma_{2}\right)^{*}$. Lemma 5.1 ensures that if $\sigma^{j}\left(G_{j}\right) \in \mathcal{A}$ for $j=1,2$, then $\sigma\left(G_{1} \times G_{2}\right) \in \mathcal{A}$. 
Given a combing $\sigma: G \rightarrow \Sigma^{*}$, a subgroup $H \subset G$ is said to be $\sigma$-quasi-convex if there is a constant $C>0$ such that $d\left(\mu\left(\sigma(h)_{t}\right), H\right) \leq C$ for all $h \in H$ and all $t \leq|\sigma(h)|$.

The following lemma is taken from [10]. It is based on ideas of M. Gromov, S. M. Gersten and H. Short. Our first use of this lemma will be in the (linguistically trivial) case where $\mathcal{A}$ is the family of all subsets of finitely generated free monoids (in which case an $\mathcal{A}$-combing is simply a combing with the fellow-traveller property).

Lemma 1.4. Let $\mathcal{A}$ be a full abstract family of languages and let $\sigma: G \rightarrow \Sigma^{*}$ be an $\mathcal{A}$-combing.

(1) If $H \subset G$ is $\sigma$-quasi-convex, then it is $\mathcal{A}$-combable; in particular it is finitely presented. If $\sigma$ is a bicombing, then $H$ is $\mathcal{A}$-bicombable. Moreover, if $\mathcal{A}=$ Reg then $H \hookrightarrow G$ is a quasi-isometric embedding with respect to any choice of word metrics.

(2) If $H_{1}, H_{2} \subset G$ are $\sigma$-quasi-convex, then so is $H_{1} \cap H_{2}$.

(3) If $\sigma$ is an $\mathcal{A}$-bicombing, then the centralizer of every $g \in G$ is $\sigma$-quasiconvex.

\subsection{Subgroups that cannot be quasi-convex}

It follows from part (1) of the previous lemma that if a subgroup $H \subset G$ is not itself $\mathcal{A}$-combable then it cannot be $\sigma$-quasi-convex with respect to any $\mathcal{A}$-combing of $G$. Also, if $G$ is automatic and $H \subset G$ is not quasi-isometrically embedded, then $H$ cannot be quasi-convex with respect to any automatic structure. Parts (2) and (3) of the lemma can be used to derive more subtle obstructions to quasi-convexity, as we shall now explain.

Proposition 1.5. Let $G$ and $Q$ be finitely generated groups. Let $\phi: Q \rightarrow \operatorname{Aut}(G)$ be a homomorphism whose kernel is not bicombable. Then:

(1) $Q \subset G \rtimes_{\phi} Q$ is not quasi-convex with respect to any bicombing of $G \rtimes_{\phi} Q$.

(2) If $\phi$ factorizes as $Q \rightarrow G \rightarrow \operatorname{Inn}(G) \subset \operatorname{Aut}(G)$, then $G \rtimes_{\phi} Q \cong G \times Q$, and in this case if $G$ and $Q$ are $\mathcal{A}$-bicombable then $G \rtimes_{\phi} Q$ is also.

Proof. The centralizer of $G$ in $G \rtimes_{\phi} Q$ is the intersection of the centralizers of the generators of $G$, which by parts (2) and (3) of the above lemma is quasiconvex with respect to any bicombing of $G \rtimes_{\phi} Q$. The intersection of $Q$ with this centralizer is the kernel of $\phi$, and by hypothesis this is not bicombable. Therefore, by part (2) of the above lemma, $Q$ cannot be quasi-convex with respect to any bicombing of $G \rtimes_{\phi} Q$.

Assume that $\phi$ factorizes as is (2), through $\phi_{0}: Q \rightarrow G$. It is easy to $\operatorname{check}^{1}$ that

\footnotetext{
$\overline{1}$ By definition, in $G \rtimes_{\phi} Q$ we have $q g q^{-1}=\phi_{0}(q) g \phi_{0}(q)^{-1}$ for all $g \in G$ and $q \in Q$.
} 
$G \rtimes_{\phi} Q$ is then the direct product of $G=G \times\{1\}$ and $\bar{Q}=\left\{\phi_{0}(q)^{-1} q \mid q \in Q\right\} \cong Q$, hence is $\mathcal{A}$-bicombable.

Remarks 1.6. (1) Similar reasoning shows that if the kernel of $\phi$ is not quasiisometrically embedded in $Q$, then $Q$ cannot be quasi-convex with respect to any biautomatic structure on $G \rtimes_{\phi} Q$.

(2) Note that the subgroup $Q \subset G \rtimes Q$ is a retract and hence is quasiisometrically embedded.

Example 1.7. Let $F$ be a finitely generated free group of rank $n$. Let $G$ be a bicombable group and let $H \subset \operatorname{Aut}(G)$ be an infinite group that is not free of rank $n$. Let $\phi: F \rightarrow H$ be a surjection. Then, by part (1) of the proposition, $F$ is not quasi-convex with respect to any bicombing of $G \rtimes_{\phi} F$, because the kernel of $\phi$ is not finitely generated.

In particular, if $G$ has no centre (so $G=\operatorname{Inn}(G)$ ), taking $H \subset \operatorname{Inn}(G)$ and appealing to 1.6(2), we obtain examples of quasi-isometrically embedded free subgroups of $G \times F \cong G \rtimes_{\phi} F$ that cannot be quasi-convex with respect to any bicombing (cf. 2.5).

\section{Groups that are not bicombable}

We shall use the properties of centralizers described in Lemma 1.4 to show that certain groups are not bicombable. The following general observations are useful in this regard.

Notation. Given a group $G$ and a subgroup $H$, we write $G \dot{*}_{H}$ to denote the trivial HNN extension $(G, t \mid[t, h]=1 \forall h \in H)$. We write $C_{G}(S)$ to denote the centralizer of $S \subset G$, and $Z(G)$ to denote the centre of $G$.

Lemma 2.1. For $i=1,2$, let $\phi_{i}: Q \rightarrow \operatorname{Aut}\left(N_{i}\right)$ be a homomorphism. Let $\phi:$ $Q \rightarrow \operatorname{Aut}\left(N_{1} * N_{2}\right)$ be the induced homomorphism (written $q \mapsto \phi_{q}$ ), and let $P=\left(N_{1} * N_{2}\right) \rtimes_{\phi} Q$. If the groups $N_{1}$ and $N_{2}$ are both non-trivial, then $C_{P}\left(N_{1} *\right.$ $\left.N_{2}\right)=\operatorname{ker} \phi=\operatorname{ker} \phi_{1} \cap \operatorname{ker} \phi_{2}$.

Proof. Each $\gamma \in P$ can be written uniquely in the form $\gamma=w q$ with $q \in Q$ and $w \in N_{1} * N_{2}$. If $\gamma \in C_{P}\left(N_{1} * N_{2}\right)$ then for $i=1,2$ and all $n \in N_{i}$ we have $n=\gamma n \gamma^{-1}=w \phi_{q}(n) w^{-1}$, hence $w^{-1} n w=\phi_{q}(n) \in N_{i}$. But $w^{-1} n w \in N_{i}$ implies $w \in N_{i}$. Hence $w \in N_{1} \cap N_{2}=\{1\}$. Therefore $\phi_{q}(n)=n$ for all $n \in N_{1} \cup N_{2}$, and $\gamma=q \in \operatorname{ker} \phi$.

Lemma 2.2. In any $H N N$ extension of the form $\Gamma=\left(N \rtimes_{\psi} H, t \mid[t, h]=1 \forall h \in\right.$ $H)$, one has $C_{\Gamma}(N) \cap C_{\Gamma}(t)=\operatorname{ker} \psi$. 
Proof. It follows immediately from Britton's Lemma that $C_{\Gamma}(t)=H \times\langle t\rangle$, and that $t^{i} N t^{-i} \cap N=\{1\}$ if $i \neq 0$.

Proposition 2.3. For $i=1,2$, let $\phi_{i}: Q \rightarrow \operatorname{Aut}\left(G_{i}\right)$ be a homomorphism, with $G_{i}$ finitely generated and non-trivial. Let $\Gamma_{i}=G_{i} \rtimes_{\phi_{i}} Q$.

(1) If $\operatorname{ker} \phi_{1} \cap$ ker $\phi_{2}$ is not bicombable (e.g. if it is not finitely presented), then $\Gamma_{1} *_{Q} \Gamma_{2}$ is not bicombable.

(2) If ker $\phi_{i}$ is not bicombable, then the trivial HNN extension $\Gamma_{i} \dot{*}_{Q}$ is not bicombable.

Proof. $\Gamma_{1} *_{Q} \Gamma_{2}=\left(G_{1} * G_{2}\right) \rtimes_{\phi} Q$ where $\phi: Q \rightarrow \operatorname{Aut}\left(G_{1} * G_{2}\right)$ is the map induced by $\phi_{1}$ and $\phi_{2}$. Thus $C\left(G_{1} * G_{2}\right)$ is not bicombable, by Lemma 2.1, and hence $\Gamma_{1} *_{Q} \Gamma_{2}$ is not bicombable, by Lemma 1.4.

The second part of the proposition follows from Lemmas 2.2 and 1.4.

Corollary 2.4. Let $G$ and $Q$ be groups and let $g \in G$ be such that no power of $g$ is central. Let $h: Q \rightarrow \mathbb{Z}$ be a homomorphism whose kernel is not bicombable. Let $\hat{Q} \subset G \times Q$ be the subgroup $\left\{\hat{q}=\left(g^{-h(q)}, q\right) \mid q \in Q\right\}$. (Note $\hat{Q} \cong Q$.) Then,

(1) the double $(G \times Q) * \hat{Q}(G \times Q)$ is not bicombable, and

(2) the trivial HNN extension $(G \times Q) \dot{*}_{\hat{Q}}$ is not bicombable.

Proof. As in the proof of Proposition 1.5, we note that $G \times Q \cong G \rtimes_{\phi} \hat{Q}$, where $\phi: \hat{Q} \rightarrow \operatorname{Aut}(G)$ takes $\hat{q}$ to the inner automorphism by $g^{-h(q)}$. Since $g^{n}$ is not central in $G$ if $n \neq 0$, we have $\operatorname{ker} \phi=\operatorname{ker} h$, and we are in the setting of the proposition, ${ }^{2}$ with $Q$ replaced by $\hat{Q}$, with $G=G_{1}=G_{2}$, and with $\phi=\phi_{1}=\phi_{2}$.

In order to animate the preceding result with examples, we seek biautomatic groups that admit surjections $h: Q \rightarrow \mathbb{Z}$ whose kernels are not bicombable. Fortunately, we do not have to look far. For example, if $Q$ is a finitely generated non-abelian free group, or surface group, then the kernel of an epimorphism $h$ : $Q \rightarrow \mathbb{Z}$ will never be finitely generated. And if $Q$ is a direct product of finitely many such free and surface groups, then the kernel of $h$ will not be of type $F P_{\infty}$ (and hence will not be bicombable). Other interesting examples come from the study of Artin groups [5] and the Rips construction [28].

In the case where $Q$ is free, as a special case of the preceding corollary we have:

Corollary 2.5. If $F$ is a non-abelian free group and $G$ is a group that contains an element $g$ no power of which is central, then $\Gamma=G \times F$ contains a subgroup $\hat{F} \cong F$ with $\Gamma=G \rtimes \hat{F}$ such that neither $\Gamma *_{\hat{F}} \Gamma$ nor $\Gamma \dot{*}_{\hat{F}}$ is bicombable.

Example 2.6. The presentation displayed in the Introduction describes the group

$\overline{2}$ or else the retract $G$ is not finitely generated 
obtained by applying Corollary 2.4(1) to the free groups $G=F(a, b)$ and $Q=$ $F(s, t)$, taking $g=a$ and $h(s)=0, h(t)=1$.

\section{Combining combings and dealing with laggards}

We shall be considering groups of the form $\Gamma=\left(N_{1} * N_{2}\right) \rtimes Q$ and combings of the form

$$
\sigma(\gamma)=\sigma^{1}(q) \sigma^{1}\left(n_{1,1}\right) \sigma^{2}\left(n_{2,1}\right) \cdots \sigma^{1}\left(n_{1, m}\right) \sigma^{2}\left(n_{2, m}\right),
$$

where $\gamma=q n_{1,1} n_{2,1} \cdots n_{1, m} n_{2, m}$ with $q \in Q$ and $n_{i, j} \in N_{i}$, and where $\sigma^{i}$ is a given combing on $N_{i} \rtimes Q$ (see Theorem 3.4). When comparing combing lines to adjacent vertices $\gamma$ and $\gamma^{\prime}=\gamma s$ in $\Gamma$, where $s \in Q$ is a generator, we are faced with the picture shown in Figure 1.

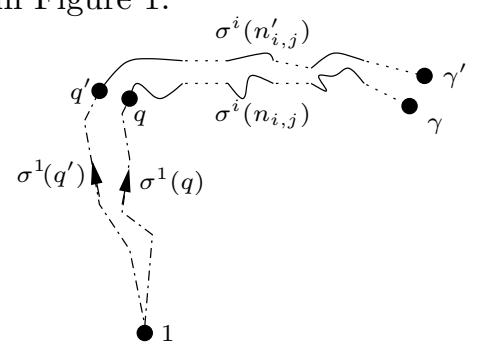

Figure 1: A combing that may suffer from laggards.

If we assume that $\sigma^{1}$ and $\sigma^{2}$ are bicombings, then the basic subpaths of $\sigma(\gamma)$ and $\sigma\left(\gamma^{\prime}\right)$ labelled $\sigma^{i}\left(n_{i, j}\right)$ and $\sigma^{i}\left(n_{i, j}^{\prime}\right)$ synchronously fellow-travel - condition (1.2). However, the lengths of this pair of basic subpaths need not be the same, and this can result in "time-lags" that may accumulate as a result of concatenation, causing $\sigma_{\gamma}$ and $\sigma_{\gamma^{\prime}}$ to be only asynchronous fellow-travellers.

One can solve this time-lag problem by slowing the paths concerned in a controlled manner. Since the time-lag problem and its solution are essentially metric phenomena, we shall phrase them in the language of metric spaces. In order to do so, we need some definitions that describe those paths in arbitrary metric spaces that behave like the paths in discrete groups defined by words in a finite generating set.

By a path in a metric space $X$ we shall mean a map $p:\left[0, T_{p}\right] \rightarrow X$. It is convenient to define $p(t)=p\left(T_{p}\right)$ for $t \geq T_{p}$ and to write $p(\infty)$ for the terminus $p\left(T_{p}\right)$. The synchronous distance between two paths can then be defined as $D\left(p_{1}, p_{2}\right):=\sup _{t} d\left(p_{1}(t), p_{2}(t)\right)$.

Let $k>0$ be a constant. We say that $p$ has speed $\leq k$ if $d\left(p(t), p\left(t^{\prime}\right)\right) \leq k\left|t-t^{\prime}\right|$ for all $t, t^{\prime}$. Given a constant $\mu \geq 1$ we write $\frac{1}{\mu} p$ to denote the path $\left[0, \mu T_{p}\right] \rightarrow X$ defined by $\frac{1}{\mu} p(t):=p(t / \mu)$. In particular, $T_{\frac{1}{\mu} p}=\mu T_{p}$.

The concatenation of two paths $p_{1}:\left[0, T_{p_{1}}\right] \rightarrow X$ and $p_{2}:\left[0, T_{p_{2}}\right] \rightarrow X$ is defined if $p_{1}(\infty)=p_{2}(0)$; it is the path $p_{1} \cdot p_{2}=\left[0, T_{p_{1}}+T_{p_{2}}\right] \rightarrow X$ defined by $p_{1} \cdot p_{2}(t)=p_{1}(t)$ if $t \leq T_{p_{1}}$ and $p_{1} \cdot p_{2}(t):=p_{2}\left(t-T_{p_{1}}\right)$ if $t \geq T_{p_{1}}$. 
Lemma 3.1. Fix a sequence of positive numbers $\left(k_{i}\right)$ such that $1+\sum_{j=1}^{i} k_{j} \leq$ $k_{i+1}$ for all $i \geq 1$. Let $X$ be a metric space and consider two finite sequences of paths $\left(p_{0}, \ldots, p_{n}\right)$ and $\left(p_{0}^{\prime}, \ldots, p_{n}^{\prime}\right)$, all of speed $\leq 1$, where $p_{i-1}(\infty)=p_{i}(0)$ and $p_{i-1}^{\prime}(\infty)=p_{i}^{\prime}(0)$ for $i=1, \ldots, n$. Suppose that there exist constants $K>0$ and $C \geq 2$ such that $D\left(p_{i}, p_{i}^{\prime}\right) \leq K$ and $\left|T_{p_{i}}-T_{p_{i}^{\prime}}\right| \leq C$ for $i=0, \ldots, n$. Let $\pi=p_{0} \cdot \frac{1}{k_{1}} p_{1} \cdots \frac{1}{k_{n}} p_{n}$ and let $\pi^{\prime}=p_{0}^{\prime} \cdot \frac{1}{k_{1}} p_{1}^{\prime} \cdots \cdots \frac{1}{k_{n}} p_{n}^{\prime}$. Then

$$
D\left(\pi, \pi^{\prime}\right) \leq K+C
$$

Proof. For $i=0, \ldots, n$ the path $\pi$ reaches the terminal point of its initial segment $P_{i}:=p_{0} \cdots \frac{1}{k_{i}} p_{i}$ at time $T(i):=T_{p_{0}}+k_{1} T_{p_{1}}+\cdots+k_{i} T_{p_{i}}$, whereas $\pi^{\prime}$ reaches the terminal point of its initial segment $P_{i}^{\prime}:=p_{0}^{\prime} \cdots \cdots \frac{1}{k_{i}} p_{i}^{\prime}$ at time $T(i)^{\prime}:=$ $T_{p_{0}^{\prime}}+k_{1} T_{p_{1}^{\prime}}+\cdots+k_{i} T_{p_{i}^{\prime}}$. From the definitions of $C$ and $\left(k_{i}\right)^{2}$ we have

$$
\delta_{i+1}:=\left|T(i)-T(i)^{\prime}\right| \leq C\left(1+k_{1}+\cdots+k_{i}\right)<C k_{i+1} .
$$

The idea of the proof is as follows. Suppose $\pi$ reached the terminus of $p_{i-1}$ before $\pi^{\prime}$ reached the terminus of $p_{i-1}^{\prime}$ and suppose that we reparameterized $\pi$ so that it then waited until $\pi^{\prime}$ caught-up before embarking on $p_{i}$, at the same time as $\pi^{\prime}$ embarked on $p_{i}^{\prime}$. If we adjusted the parameterizations of $\pi$ and $\pi^{\prime}$ in this manner at the termini of all of the subpaths $p_{j}$ and $p_{j}^{\prime}$, then it would be clear from the definitions that $\pi$ and $\pi^{\prime}$ (thus parameterized) would remain $K$-close. However, we are interested in the synchronous fellow-traveller property, not the asynchronous one, so we cannot allow $\pi$ to wait for $\pi^{\prime}$, or vice versa. It is to circumvent this problem that we have introduced the weighting factors $k_{i}$ : the inequality displayed above ensures that the difference in time between the moment when $\pi$ embarks on $p_{i}$ and the moment when $\pi^{\prime}$ embarks on $p_{i}^{\prime}$ is less than $C k_{i}$, and because we have slowed the speed of $p_{i}$ by a factor of $k_{i}$, the subpath that $\pi$ traverses in the interval before $\pi^{\prime}$ embarks on $p_{i}^{\prime}$ will have length less than $C$.

To obtain a precise estimate, we define $\tau_{i}=\max \left\{T(i-1), T(i-1)^{\prime}\right\}$ and consider $d\left(\pi(t), \pi^{\prime}(t)\right)$ with $t \in\left[\tau_{i}, \tau_{i+1}\right]$. We may assume without loss of generality that $\delta_{i}=T(i-1)^{\prime}-T(i-1) \geq 0$. Let $\bar{t}:=\frac{1}{k_{i}}\left(t-T(i-1)^{\prime}\right)$ and note that $\pi^{\prime}(t)=p_{i}^{\prime}(\bar{t})$ for $t \in\left[\tau_{i}, T(i)^{\prime}\right]$ and $\pi\left(t-\delta_{i}\right)=p_{i}(\bar{t})$ for $t \in\left[\tau_{i}, T(i)+\delta_{i}\right]$. There are three cases to consider.

Case 1 , where $t \leq \min \left\{T(i), T(i)^{\prime}\right\}$ : In this case $\pi^{\prime}(t)=p_{i}^{\prime}(\bar{t})$ and $\pi\left(t-\delta_{i}\right)=$ $p_{i}(\bar{t})$, so

$$
d\left(\pi(t), \pi^{\prime}(t)\right) \leq d\left(\pi(t), \pi\left(t-\delta_{i}\right)\right)+d\left(p_{i}(\bar{t}), p_{i}^{\prime}(\bar{t})\right) \leq C+K
$$

where the estimate $d\left(\pi(t), \pi\left(t-\delta_{i}\right)\right) \leq C$ is obtained by noting that the path $s \mapsto \pi\left(t-\delta_{i}+s\right)$, with $s \in\left[0, \delta_{i}\right]$, has speed at most $1 / k_{i}$ and hence has length at most $\delta_{i} / k_{i}$, which is less than $C$.

Case 2, where $\tau_{i+1}=T(i)^{\prime}$ and $t \in\left[T(i), T(i)^{\prime}\right]$ : If $t \leq T(i)+\delta_{i}$ then the estimate from Case 1 still pertains. If $t \geq T(i)+\delta_{i}$, then $\bar{t} \geq T_{p_{i}}$ and hence $d\left(p_{i}(\infty), p_{i}^{\prime}(\bar{t})\right) \leq K$. Therefore, noting that $\pi^{\prime}(t)=p_{i}^{\prime}(\bar{t})$ and $p_{i}(\infty)=\pi(T(i))$, 
we have

$$
d\left(\pi(t), \pi^{\prime}(t)\right) \leq d\left(\pi(t), \pi(T(i))+d\left(p_{i}(\infty), p_{i}^{\prime}(\bar{t})\right) \leq C+K,\right.
$$

where the estimate $d(\pi(t), \pi(T(i)) \leq C$ is obtained by noting that the path $s \mapsto$ $\pi(T(i)+s)$, with $s \in[0, t-T(i)] \subset\left[0, T(i)^{\prime}-T(i)\right]$, has speed bounded above by $1 / k_{i+1}$ and hence length at most $\delta_{i+1} / k_{i+1}$, which is less than $C$.

Case 3, where $\tau_{i+1}=T(i)$ and $t \in\left[T(i)^{\prime}, T(i)\right]$ : In this case $\pi(t)=p_{i}(\tilde{t})$ where $\tilde{t} \geq T_{p_{i}^{\prime}}$ and hence $d\left(p_{i}(\tilde{t}), p_{i}^{\prime}(\infty)\right) \leq K$. Noting that $p_{i}^{\prime}(\infty)=\pi^{\prime}\left(T(i)^{\prime}\right)$, we have

$$
d\left(\pi(t), \pi^{\prime}(t)\right) \leq d\left(p_{i}(\tilde{t}), p_{i}^{\prime}(\infty)\right)+d\left(\pi^{\prime}\left(T(i)^{\prime}\right), \pi^{\prime}(t)\right) \leq K+C,
$$

where the estimate $d\left(\pi^{\prime}\left(T(i)^{\prime}\right), \pi^{\prime}(t)\right) \leq C$ is obtained by noting that the path $s \mapsto \pi^{\prime}\left(T(i)^{\prime}+s\right)$, with $s \in\left[0, t-T(i)^{\prime}\right]$, has speed bounded above by $1 / k_{i+1}$ and hence length at most $\delta_{i+1} / k_{i+1}$, which is less than $C$.

Remark 3.2. In order to enhance the clarify of the exposition, we placed a rather simple condition on the scaling factors $\left(k_{i}\right)$ in the above lemma; the reader should have no difficulty in seeing that the condition can easily be weakened in a number of ways. For example, one can weaken the given condition by requiring only that there exist a constant $m>0$ such that $1+\sum_{j=1}^{i} k_{j} \leq m k_{i+1}$, and one can weaken it further by requiring only that this inequality hold for sufficiently large values of $i$. In each case the conclusion of the lemma has to be weakened slightly but its essence remains intact, namely $D\left(\pi, \pi^{\prime}\right)$ is uniformly bounded. In each case, the proof requires only the most minor of modifications.

We explained earlier how one can regard a word $w$ in the generators $\Sigma$ of a finitely generated group as discrete paths in the group. This extends to a path $\hat{w}$ of speed $\leq 1$ in the Cayley graph associated to $\Sigma$. The following device allows one to follow the preceding reparameterization arguments in this algebraic setting.

Definition 3.3. Let $k>0$ be an integer. Given a finite set $\Sigma$, a symbol $o \notin \Sigma$ and a word $w=s_{1} \ldots s_{n} \in \Sigma^{*}$, we define the word $\frac{1}{k} w \in(\Sigma \cup\{o\})^{*}$ to be

$$
\frac{1}{k} w=s_{1} o \ldots o s_{2} o \ldots o s_{3} o \ldots o s_{n} o \ldots o
$$

where there are $(k-1)$ letters $o$ following each letter $s_{i}$.

Note that the word metric $d$ on $\Gamma$ associated to the finite generating set $\Sigma$ is the same as that associated to $\Sigma \cup\{o\}$ if we define $o$ to represent $1 \in \Gamma$. Moreover, the path in the metric space $(\Gamma, d)$ defined by the word $\frac{1}{k} w$ is $\frac{1}{k} \hat{w}$ (in the metric space notation defined prior to Lemma 3.1), where $\hat{w}$ is the path in the Cayley graph extending $w$.

Theorem 3.4. If the groups $\Gamma_{1}=N_{1} \rtimes Q$ and $\Gamma_{2}=N_{2} \rtimes Q$ admit bicombings that have the comparable lengths property, then $\Gamma=\left(N_{1} * N_{2}\right) \rtimes Q=\Gamma_{1} *_{Q} \Gamma_{2}$ is combable. 
Proof. Fix a finite generating set $\mathcal{B}$ for $Q$ and extend this to finite generating sets $\Sigma_{1}=\Sigma_{1}^{-1}$ for $\Gamma_{1}$ and $\Sigma_{2}=\Sigma_{2}^{-1}$ for $\Gamma_{2}$. We may assume that no $s \in \Sigma_{i} \backslash \mathcal{B}$ evaluates to an element of $Q$. For $i=1,2$, choose bicombings $\sigma^{i}: \Gamma_{i} \rightarrow \Sigma_{i}^{*}$ and fix positive integers $C$ and $K$ so that conditions (1.2) and (1.3) hold with $\sigma=\sigma^{i}$. Note in particular that

$$
D\left(s \cdot \sigma^{i}\left(s^{-1} g s\right), \sigma^{i}(g)\right) \leq K
$$

for all $s \in \mathcal{B}$ and $g \in \Gamma_{i}$, for $i=1,2$.

Using the semi-direct and free product structures, each element $\gamma \in \Gamma$ can be written uniquely in the form

$$
\gamma=q n_{1,1} n_{2,1} \ldots n_{1, m} n_{2, m}
$$

where $q \in Q$ and where the elements $n_{i, j} \in \Gamma_{i}$ are non-trivial except possibly for $n_{1,1}$ and $n_{2, m}$. We define a combing $\sigma: \Gamma \rightarrow\left(\Sigma_{1} \cup \Sigma_{2}\right)^{*}$ by:

$$
\sigma(\gamma)=\sigma^{1}(q) \cdot \frac{1}{k} \sigma^{1}\left(n_{1,1}\right) \cdot \frac{1}{k^{2}} \sigma^{2}\left(n_{2,1}\right) \cdots \frac{1}{k^{2 m-1}} \sigma^{1}\left(n_{1, m}\right) \cdot \frac{1}{k^{2 m}} \sigma^{2}\left(n_{2, m}\right),
$$

where $k \geq 2$ is an integer.

We must show that $\sigma$ satisfies the fellow-traveller property (1.1). Viewing words as paths from $1 \in \Gamma$ in the usual way, it suffices to show that $D(\sigma(\gamma s), \sigma(\gamma)) \leq$ $K+C$ for all $\gamma \in \Gamma$ and $s \in \Sigma_{1} \cup \Sigma_{2}$.

Case 1: $s \in \mathcal{B}$. In this case

$$
\gamma s=q\left(\prod_{(i, j)=(1,1), \ldots,(2, m)} n_{i, j}\right) s=(q s) \prod_{(i, j)=(1,1), \ldots,(2, m)} s^{-1} n_{i, j} s .
$$

Therefore

$$
\begin{aligned}
\sigma(\gamma s)=\sigma^{1}(q s) \cdot \frac{1}{k} \sigma^{1}\left(s^{-1} n_{1,1} s\right) & \cdot \frac{1}{k^{2}} \sigma^{2}\left(s^{-1} n_{2,1} s\right) \\
& \cdots \\
& \cdots \frac{1}{k^{2 m-1}} \sigma^{1}\left(s^{-1} n_{1, m} s\right) \frac{1}{k^{2 m}} \sigma^{2}\left(s^{-1} n_{2, m} s\right) .
\end{aligned}
$$

If we write $p_{i, j}$ for the subpath of $\sigma(\gamma)$ corresponding to $\sigma^{i}\left(n_{i, j}\right)$, and $p_{i, j}^{\prime}$ for the subpath of $\sigma(\gamma s)$ corresponding to $\sigma^{i}\left(s^{-1} n_{i, j} s\right)$, and $P(i, j)$ and $P(i, j)^{\prime}$ for the prefixes before these subwords, then as elements of $\Gamma$ we have the equality $P(i, j)^{\prime}=P(i, j) s$, and therefore

$$
D\left(p_{i, j}, p_{i, j}^{\prime}\right)=D\left(\sigma^{i}\left(n_{i, j}\right), s . \sigma^{i}\left(s^{-1} n_{i, j} s\right)\right) \leq K
$$

(by the first inequality in this proof). We also have $D\left(\sigma^{1}(q), \sigma^{1}(q s)\right) \leq K$. Thus we are in the setting of Lemma 3.1, and hence $D(\sigma(\gamma s), \sigma(\gamma)) \leq K+\bar{C}$.

Case 2: $s \in \Sigma_{1} \backslash \mathcal{B}$. If $n_{2, m} \neq 1$, then $\sigma(\gamma s)$ is obtained from $\sigma(\gamma)$ simply by appending $\frac{1}{k^{2 m+1}} s$, and thus $D(\sigma(\gamma s), \sigma(\gamma))=1$. If $n_{2, m}=1$, then $\sigma(\gamma s)$ is obtained from $\sigma(\gamma)$ by replacing the terminal segment $\frac{1}{k^{2 m-1}} \sigma^{1}\left(n_{1, m}\right)$ with $\frac{1}{k^{2 m-1}} \sigma^{1}\left(n_{1, m} s\right)$, and hence $D(\sigma(\gamma s), \sigma(\gamma)) \leq K$.

Case 3: $s \in \Sigma_{2} \backslash \mathcal{B}$. Entirely similar to case 2 . 
Remarks 3.5. (1) In the above theorem there is no assumption that either $Q$ or $N_{i}$ is quasi-convex. Indeed there are many interesting examples where the $N_{i}$ are not even finitely generated.

(2) In general $\Gamma_{1} *_{Q} \Gamma_{2}$ will not be bicombable (see below).

(3) In the above construction one could replace $\left.\sigma\right|_{Q}=\left.\sigma^{1}\right|_{Q}$ by any combing of $Q$ that has the fellow-traveller and comparable lengths properties - the proof would work without alteration. Thus the combing on $Q$ need not be related to $\sigma^{1}$ or $\sigma^{2}$ in any way.

(4) There is a good deal of flexibility in how one parameterizes the combing lines in the above proof: for convenience we used scaling factors $k^{i}$, but our appeal to Lemma 3.1 would have remained valid is we had used any sequence $\left(k_{i}\right)$ for which there is a constant $m>0$ such that $1+\sum_{j=1}^{i} k_{j} \leq m k_{i+1}$ (cf. Remark 3.2 ). In particular, given an integer $k \geq 2$, we could have taken $k_{i}=k^{i}+1$. This observation will be useful in Section 6 .

In the light of Lemma 1.3(1), as special cases of the above theorem we have:

\section{Corollary 3.6.}

(1) Let $Q$ be a finitely generated group. If $\Gamma_{1}=N_{1} \rtimes Q$ and $\Gamma_{2}=N_{2} \rtimes Q$ are biautomatic, then $\Gamma_{1} *_{Q} \Gamma_{2}$ is combable.

(2) If $Q$ and $G=N \rtimes Q$ are biautomatic, then the trivial $H N N$ extension $G \dot{*}_{Q}$ is combable.

Proof. For (2), note that $G \dot{*}_{Q}=(N \rtimes Q) *_{Q}(\mathbb{Z} \times Q)$.

\section{Proof of Theorems A and B}

By combining 3.4 and 2.3 we obtain the main technical result behind Theorems A and B:

Main Lemma. If the groups $\Gamma_{1}=N_{1} \rtimes_{\phi_{1}} Q$ and $\Gamma_{2}=N_{2} \rtimes_{\phi_{2}} Q$ admit bicombings that have the comparable lengths property, then $\Gamma=\Gamma_{1} *_{Q} \Gamma_{2}$ is combable. But for finitely generated $N_{i}$ if $\operatorname{ker} \phi_{1} \cap \operatorname{ker} \phi_{2}$ is not bicombable, then neither is $\Gamma$.

We claim that there are many groups that satisfy the hypotheses of both parts of this lemma, and hence Theorem A follows from it. In order to obtain a large class of examples, we combine Corollaries 2.5 and 3.6 and refine them in the following manner:

Theorem 4.1. Let $G$ be a biautomatic group and let $F$ be a finitely generated nonabelian free group. If $G$ modulo its centre is infinite, then $\Gamma_{0}:=G \times F$ contains a subgroup $\hat{F} \cong F$ with $\Gamma_{0}=G \rtimes \hat{F}$ such that: 
(1) $\Gamma_{0} *_{\hat{F}} \Gamma_{0}$ and $\Gamma_{0} \dot{*}_{\hat{F}}$ are combable, but

(2) neither $\Gamma_{0} *_{\hat{F}} \Gamma_{0}$ nor $\Gamma_{0} \dot{*}_{\hat{F}}$ is bicombable.

Proof. If we assume that $G$ contains an element $g$ no power of which is central, then the theorem follows immediately from 2.5 and 3.6. If $G$ does not contain such an element $g$, then $G / Z(G)$ would be an infinite torsion group. But if a biautomatic group is infinite then it contains an element of infinite order [18], and Mosher proves that $G / Z(G)$ is biautomatic [23].

We recalled the definition of the Dehn function of a finite presentation before stating Lemma 1.2.

Proof of Theorem B. Let $F$ be a finitely generated non-abelian free group. By taking $G=F$ in the preceding theorem we obtain combable groups $(F \times F) \dot{*} \hat{F}$ and $(F \times F) *_{\hat{F}}(F \times F)$. The Dehn functions of both of these groups are cubic this is proved on page 504 of [12] by a direct analysis of van Kampen diagrams. Moreover, a simple adaptation of the arguments presented there shows that all of the groups described in Theorem 4.1 have cubic Dehn functions.

Automatic groups satisfy a quadratic isoperimetric inequality (see Lemma 1.2), so the preceding proof yields:

Corollary 4.2. The combable groups described in Theorem 4.1 are not automatic.

Remark 4.3. It is still not known whether all automatic groups are biautomatic. Nor is it known whether all groups that admit a synchronous combing by quasigeodesics are bicombable.

\section{Full abstract families of languages}

In the next section we shall analyse the grammatical complexity of the combings constructed in Theorem 4.1. In preparation for this, in this section we recall some basic facts about formal languages, i.e. subsets of finitely generated free monoids. Our discussion follows that of [10]. We refer the reader to Hopcroft and Ullman [22] for an introduction to formal language theory, and to Epstein et al. [15] for a thorough account of how the study of such languages came to play an important role in group theory and geometry/topology.

Given a set (alphabet) $\Sigma$, we write $\Sigma^{*}$ to denote the free monoid over $\Sigma$. As in Section 1, we shall implicitly assume that $\Sigma$ is finite. The identity element of $\Sigma^{*}$ is the empty word $\epsilon$, and multiplication of words is by concatenation. Given $L \subseteq \Sigma^{*}$, we let $L^{*}$ denote the submonoid of $\Sigma^{*}$ generated by $L$. If $L, M \subseteq \Sigma^{*}$, then $L M=\{w \mid w=x y, x \in L, y \in M\}$ is the product of $L$ and $M$. If $L \subseteq \Sigma^{*}$, then $\Sigma$ is called an alphabet for $L$, and $L$ is called a language over $\Sigma$. 
We will use regular, context-free, and indexed languages. These classes of languages form an increasing hierarchy. We consider these classes simultaneously as instances of a full abstract family of languages. A full AFL is a class of languages which contains a non-empty language and is closed under the operations listed in part (1) of the following lemma. See [22] Chapter 11 for more details.

Lemma 5.1. Let $\mathcal{A}$ be a full $A F L$.

(1) $\mathcal{A}$ is closed under homomorphism (i.e., image under homomorphism of the ambient free monoid to another finitely generated free monoid), inverse homomorphism (likewise), intersection with regular sets, union, product, and generation of submonoid.

(2) $\mathcal{A}$ contains all regular languages.

(3) If $L_{1}$ and $L_{2}$ are both in $\mathcal{A}$, then the language $L_{1} \bullet L_{2}$ consisting of $\epsilon$ and all words $w=u_{1} \ldots u_{n}$ with successive $u_{i}$ chosen alternately from $L_{1}$ and $L_{2}$ is also in the $\mathcal{A}$. (The notation $L_{1} \bullet L_{2}$ is not standard, but we shall use it consistently.)

(4) The classes of regular, context-free, and indexed languages are each a full AFL.

Proof. Part (1) is true by definition; for (2) see [22] and for (4) see [22] and [1]. Finally the language $L_{1} \bullet L_{2}$ in (3) can be constructed from $L_{1}$ and $L_{2}$ by the operations given in (i): it is $\left(L_{1} L_{2}\right)^{*} \cup\left(L_{1} L_{2}\right)^{*} L_{1} \cup\left(L_{2} L_{1}\right)^{*} \cup\left(L_{2} L_{1}\right)^{*} L_{2}$.

\section{Grammars}

Regular, context-free and indexed languages are generated by corresponding types of grammars (see [22]). Briefly, an indexed grammar has disjoint finite sets $N$, $\Sigma, F$ of nonterminals, terminals, and indices respectively. The language (over $\Sigma$ ) generated by the grammar is obtained by beginning with a designated start symbol $S \in N$ and performing substitutions. The allowed substitutions are determined by a finite set of productions, and the grammar is completely described by $N, \Sigma, F$, $S$, and the set of productions. Productions of three types are allowed: (1) $A \rightarrow \alpha$, (2) $A \rightarrow B f$, (3) $A f \rightarrow \alpha$, where $A$ and $B$ are nonterminals, $\alpha \in(N \cup \Sigma)^{*}$, and $f$ is an index. Roughly speaking (we shall explain more precisely in a moment) the corresponding substitutions consist of replacing the left-hand side of a production by the right-hand side. This procedure generates words in $(N \cup \Sigma \cup F)^{*}$. The words in $\Sigma^{*}$ which can be derived in this way form the language generated by the grammar.

Words in $(N \cup \Sigma \cup F)^{*}$ are called sentential forms. To apply a production of type (1) to a sentential form $\beta$ one finds an occurrence of $A$ in $\beta$ and substitutes $\alpha$ for $A$. The string of indices $\delta$ (possibly the empty string) following the occurrence of $A$ in $\beta$ is repeated after each nonterminal in $\alpha$ (there is never a string of indices 
following a terminal). In case (2) one simply substitutes $B f$ for $A$. In case (3) one finds an occurrence of $A f$ followed by a string of indices $\delta$, substitutes $\alpha$ for $A f$, and repeats $\delta$ after each nonterminal in $\alpha$.

If a sentential form $\beta$ can be obtained from a sentential form $\alpha$ by a finite sequence of productions, then one writes $\alpha \stackrel{*}{\rightarrow} \beta$.

A context-free grammar is the same as an indexed grammar except that there are no indices, and a regular grammar is a context-free grammar in which all productions have a right-hand side consisting of an element of $\Sigma \cup\{\epsilon\}$ possibly followed by a single nonterminal.

Example 5.2. An example of a context-free language that is not regular is $\left\{a^{n} b^{n} \mid\right.$ $n \in \mathbb{N}\}$ and an example of an indexed language that is not context-free is $\left\{a^{n} b^{n^{2}}\right.$ $n \in \mathbb{N}\}$ (see [10], [19] and the next section for more interesting examples). An example of a context sensitive language that is not indexed is $\left\{\left(a b^{n}\right)^{n} \mid n \in \mathbb{N}\right\}$.

Remark 5.3. Indexed languages enjoy the following "context-freeness": if $\alpha$ and $\beta$ are sentential forms for an indexed grammar and $\alpha$ does not end with an index, then $\{\gamma \mid \alpha \beta \stackrel{*}{\rightarrow} \gamma\}=\left\{\gamma_{1} \gamma_{2} \mid \alpha \stackrel{*}{\rightarrow} \gamma_{1}, \beta \stackrel{*}{\rightarrow} \gamma_{2}\right\}$. Moreover, one may factorize $\alpha \beta \stackrel{*}{\rightarrow} \gamma_{1} \gamma_{2}$ as either $\alpha \beta \stackrel{*}{\rightarrow} \gamma_{1} \beta \stackrel{*}{\rightarrow} \gamma_{1} \gamma_{2}$ or $\alpha \beta \stackrel{*}{\rightarrow} \alpha \gamma_{2} \stackrel{*}{\rightarrow} \gamma_{1} \gamma_{2}$.

A celebrated theorem of Muller and Schupp [24] (which relies on Dunwoody's Accessibility Theorem [14]) states that, given a finite generating set for a group $G$, the set of words representing the identity in $G$ is a context-free language if and only if $G$ contains a free subgroup of finite index. We need a special case of (the easier direction of) this result:

Lemma 5.4. If $F$ is a free group with finite generating set $\Sigma=\Sigma^{-1}$, then the set of words in $\Sigma^{*}$ that represent the identity in $F$ is a context-free language.

Corollary 5.5. Let $G$ be a group with a finite set of generators $\mu: \Sigma^{*} \rightarrow G$. Let $L \subset \Sigma^{*}$ be a regular language. Let $\phi: G \rightarrow F$ be an epimorphism to a finitely generated free group. Then $\{w \in L \mid \mu(w) \in$ ker $\phi\}$ is a context-free language.

Proof. According to the lemma, the language $\left\{w \in \Sigma^{*} \mid \phi \circ \mu(w)=1\right\}$ is contextfree. The intersection of a context-free language and a regular language is always context-free (see 5.1).

\section{The grammar of reparameterization}

In this section we shall prove:

Theorem D. There exist Ind-combable groups that are not Reg-combable (i.e. automatic). 
Indexed languages enter our discussion naturally for the following reason: in order to construct the combings that we needed in our proof of Theorems A and B, we had to "slow-down" certain combings that lacked the synchronous fellowtraveller property. In other words, we redefined the combing in such a way that the discrete path that each combing line traced through the group was left unaltered but the speed at which it traversed this path was reduced (see 3.3). We shall now explore the effect that this slowing-down has on the grammatical complexity of the language of words in the combing. In particular, assuming that the original language was generated by a certain grammar, we shall explain how to modify the grammar so as to slow the combing in the desired manner. This modification is achieved by mingling the original grammar with a certain indexed grammar; if the original grammar was regular or context-free, then the final grammar will be indexed, and hence the image of the final combing will an indexed language.

Definition 6.1. Let $k>0$ be an integer. Given a finite set $\Sigma$, a symbol $o \notin \Sigma$ and languages $L_{1}, L_{2} \subset \Sigma^{*}$, we define $\left[\frac{1}{k}\right] L_{1} \bullet L_{2}$ to be the sub-language of $(\Sigma \cup\{o\})^{*}$ consisting of the empty word and

$$
\left\{w_{0} \frac{1}{k_{1}} w_{1} \ldots \frac{1}{k_{m}} w_{m} \mid m \in \mathbb{N}, w_{i} \text { taken alternately from } L_{1} \text { and } L_{2}\right\}
$$

where $k_{i}=k^{i}+1$ (notation of 3.3). Thus a word in $\left[\frac{1}{k}\right] L_{1} \bullet L_{2}$ is obtained from a word $w_{0} \ldots w_{m} \in L_{1} \bullet L_{2}$ by introducing a string of $k^{i}$ letters $o$ after each letter in the subword $w_{i}$ for $i=1, \ldots, m$.

\section{The indexed grammar of reparameterization}

Consider the indexed grammar with initial state $S$ that has non-terminals $A_{1}, A_{2}$, $X_{1}, X_{2}, Y_{1}, Y_{2}, S_{1}, S_{2}, I$, terminals $x_{1}, x_{2}, i$, two indices $f, g$, and productions

$$
\begin{aligned}
S & \rightarrow X_{1} g \text { or } X_{2} g \text { or } X_{1} g A_{2} g \text { or } X_{2} g A_{1} g \\
A_{1} & \rightarrow Y_{1} f ; \quad A_{2} \rightarrow Y_{2} f \\
Y_{1} & \rightarrow X_{1} \text { or } X_{1} A_{2} ; \quad Y_{2} \rightarrow X_{2} \text { or } X_{2} A_{1} \\
X_{1} g & \rightarrow x_{1} ; \quad X_{2} g \rightarrow x_{2} ; \\
X_{1} f & \rightarrow x_{1} I ; \quad X_{2} f \rightarrow x_{2} I ; \quad \text { If } \rightarrow i I \quad I g \rightarrow i .
\end{aligned}
$$

If we were to delete from this grammar the indices $f$ and $g$ and the symbol $I$, then we would be left with a context-free grammar generating the language of all words of the form $x_{1} x_{2} x_{1} x_{2} \ldots$ and $x_{2} x_{1} x_{2} x_{1} \ldots$. The effect of introducing the indices and $I$ is to place a string of letters $i$ after each letter of each word in the language just described: the string after the $m$-th letter of each word is $(m-1)$ letters long. Thus the shortest words in the language are:

$x_{1}, x_{2}, x_{1} x_{2} i, x_{2} x_{1} i, x_{1} x_{2} i x_{1} i i, x_{2} x_{1} i x_{2} i i, x_{1} x_{2} i x_{1} i i x_{2} i i i, x_{2} x_{1} i x_{2} i i x_{1} i i i, \ldots$ 
For example,

$$
\begin{aligned}
S & \rightarrow X_{1} g A_{2} g \rightarrow x_{1} A_{2} g \rightarrow x_{1} Y_{2} f g \rightarrow x_{1} X_{2} f g A_{1} f g \rightarrow x_{1} X_{2} f g Y_{1} f^{2} g \rightarrow \\
& \rightarrow x_{1} X_{2} f g X_{1} f^{2} g \rightarrow x_{1} x_{2} I g X_{1} f^{2} g \rightarrow x_{1} x_{2} I g x_{1} I f g \rightarrow \\
& \rightarrow x_{1} x_{2} I g x_{1} i I g \rightarrow x_{1} x_{2} I g x_{1} i i \rightarrow x_{1} x_{2} i x_{1} i i .
\end{aligned}
$$

Remark 6.2. There is an obvious simplification of the above grammar: one could replace the $X_{j} g$ in the top row by $x_{j}$ and remove the productions in the penultimate row. We have chosen the more clumsy structure because it lends itself readily to the following application.

Theorem 6.3. Let $\Sigma$ be a finite set and let $k>0$ be an integer. If $L_{1}, L_{2} \subset \Sigma^{*}$ are context-free languages, then $L:=\left[\frac{1}{k}\right] L_{1} \bullet L_{2}$ is an indexed language.

Proof. Let $\mathcal{G}_{1}$ and $\mathcal{G}_{2}$ be context-free grammars over the alphabet $\Sigma$, generating the languages $L_{1}$ and $L_{2}$ respectively. We shall generate $L$ by an indexed grammar over the alphabet $\Sigma \cup\{o\}$, where the symbol $o$ is as in the definition of $\left[\frac{1}{k}\right] L_{1} \bullet L_{2}$.

Let $S$ denote the start symbol of our grammar. The grammar has two indices $f$ and $g$. The non-terminals of the grammar are of five kinds: the non-terminals of $\mathcal{G}_{1}$ and $\mathcal{G}_{2}$; the symbols $S_{1}$ and $S_{2}$ that were previously regarded as the start symbols of $\mathcal{G}_{1}$ and $\mathcal{G}_{2}$; one symbol $B$ for each terminal $b \in \Sigma$; four new symbols $A_{1}, A_{2}, Y_{1}, Y_{2}$ (that will perform as they did in the grammar of reparameterization described prior to this theorem); and one other symbol $O$. We group the productions into four types: we have the productions listed in the first three rows of the grammar of reparameterization, modified by replacing $X_{1}$ and $X_{2}$ by $S_{1}$ and $S_{2}$ respectively; we also have the productions from the grammars $\mathcal{G}_{1}$ and $\mathcal{G}_{2}$, which are unaltered except for the fact that every occurrence of $b \in \Sigma$ is replaced by $B$; we have two additional productions for each $b \in \Sigma$, namely

$$
B f \rightarrow b O \ldots O \text { and } B g \rightarrow b,
$$

where there are $k$ letters $O$ after the $b$ in the first production; and finally we have the productions

$$
O f \rightarrow O \ldots O \text { and } O g \rightarrow o,
$$

where there are $k$ letters $O$ on the right of the first production.

It follows from remark 5.3 that a word $w \in(\Sigma \cup\{o\})^{*}$ lies in the language generated by the above grammar if and only if $w$ can be obtained by applying a sequence of productions from the (modified) grammar of reparameterization, followed by a sequence of productions from the (modified) grammars $\mathcal{G}_{j}$, followed by instances of the last two types of productions displayed above. Thus we may assume that

$$
S \stackrel{*}{\rightarrow} \alpha \stackrel{*}{\rightarrow} \beta \stackrel{*}{\rightarrow} w,
$$

where the sentential form $\alpha$ is a word in the symbols $\left\{S_{1}, S_{2}, g, f\right\}$, where $\beta$ is a word in $f, g$ and the various symbols $B$, and where $\beta \stackrel{*}{\rightarrow} w$ involves only the two 
new types of production (the $B g \rightarrow b$ and those involving $O$ ).

The productions in the first three rows of the grammar of reparameterization generate the following sentential forms in $\left\{X_{1}, X_{2}, g, f\right\}$ :

$$
X_{1} g X_{2} f g X_{1} f^{2} g X_{2} f^{3} g \ldots X_{1} f^{2 r} g \text { and } X_{1} g X_{2} f g X_{1} f^{2} g X_{2} f^{3} g \ldots X_{2} f^{2 r+1} g
$$

( $r$ any non-negative integer) and the same forms with the roles of $X_{1}$ and $X_{2}$ reversed. The form $\alpha$ is obtained from such a form by making the substitutions $X_{j} \rightarrow S_{j}$.

One proceeds from $\alpha$ to $\beta$ by applying a sequence of productions from the (modified) context-free grammars $\mathcal{G}_{j}$ to each of the non-terminals in $\alpha$. (The sequences of productions applied to the different non-terminals in $\alpha$ are independent because the $\mathcal{G}_{j}$ are context-free.) An important point to note is that since we have replaced all of the terminals $b$ in the productions of $\mathcal{G}_{j}$ with non-terminals $B$, the index $f^{m} g$ appearing after a non-terminal $S_{j}$ in $\alpha$ appears after every symbol in the sentential forms derived from that $S_{j}$ in the course of passing to $\beta$. Thus $\beta$ is a product of sub-forms $B f^{m} g$. For example, if $\alpha=S_{1} g S_{2} f g S_{1} f^{2} g \ldots S_{1} f^{2 r} g$, then $\beta=U_{0} V_{1} U_{2} \ldots U_{r}$ where: every symbol in $U_{i}$ is a non-terminal $B$ followed by a string of $2 i$ indices $f$, followed by $g$; every symbol in $V_{i}$ is a non-terminal $B$ followed by a string of $2 i-1$ indices $f$, followed by $g$; the word obtained from $U_{j}$ by deleting the indices $f$ and $g$ and then replacing each $B$ by the corresponding $b \in \Sigma$ belongs to $L_{1}$; the word obtained from $V_{j}$ by deleting the indices $f$ and $g$ and then replacing each $B$ by the corresponding $b \in \Sigma$ belongs to $L_{2}$.

Finally, to realize $\beta \stackrel{*}{\rightarrow} w \in L$, to each of the sub-forms $B f^{m} g$ in $\beta$ one applies the production $B f \rightarrow b O \ldots O$ (if $m \neq 0$ ) followed by $(m-1)$ generations of productions $O f \rightarrow O \ldots O$, then (having got rid of all of the indices $f$ ) one applies the production $O g \rightarrow o$ to each $O g$ (and $B g \rightarrow b$ in the case $m=0$ ). For example,

$$
B f^{3} g \rightarrow b(O f f g)^{k} \stackrel{*}{\rightarrow} b\left((O f g)^{k}\right)^{k} \stackrel{*}{\rightarrow} b\left(\left((O g)^{k}\right)^{k}\right)^{k}=b(O g)^{k^{3}} \stackrel{*}{\rightarrow} b o^{k^{3}} .
$$

This description of $\beta \stackrel{*}{\rightarrow} w$, together with our earlier description of $\alpha$ and $\alpha \stackrel{*}{\rightarrow} \beta$, completes the proof that $S \stackrel{*}{\rightarrow} w$ if and only if $w \in\left[\frac{1}{k}\right] L_{1} \bullet L_{2}$; in other words $L$ is the indexed language generated by the indexed grammar described in the second paragraph of the proof.

Theorem D, as stated in the introduction, is an immediate consequence of 4.1, 4.2 and the following theorem.

Theorem 6.4. Let $\Gamma_{1}=N_{1} \rtimes F$ and $\Gamma_{2}=N_{2} \rtimes F$ be biautomatic groups. If $F$ is finitely generated and free, then $\Gamma=\Gamma_{1} *_{F} \Gamma_{2}$ is Ind-combable.

Proof. In the proof of 4.1 we constructed a combing $\sigma: \Gamma \rightarrow(\Sigma \cup\{o\})^{*}$. If we assume that the scaling factors used in the construction of $\sigma$ are modified as in Remark 3.5(4), then, in the language of 6.1, the image of $\sigma$ is $L_{0} \cdot\left[\frac{1}{k}\right] L_{1} \bullet L_{2}$, where $L_{0}$ can be taken to be the image of an automatic structure on $F$, and $L_{i}$ is the image of $N_{i}$ under a regular bicombing (biautomatic structure) $\sigma^{i}: \Gamma_{i} \rightarrow \Sigma^{*}$. 
Because $\sigma^{i}\left(\Gamma_{i}\right)$ is a regular language for $i=1,2$, it follows from Lemma 5.5 that $L_{1}$ and $L_{2}$ are context-free languages. But then Theorem 6.3 implies that $\left[\frac{1}{k}\right] L_{1} \bullet L_{2}$ is an indexed language. Since the class of indexed languages is closed under the formation of products, we deduce that the image of $\sigma$ is an indexed language.

Remarks 6.5. (1) When I came to write the final draft of this article, it seemed most natural to arrange it so that indexed grammars appear in a secondary role at the end. However, it was my attempts to understand the class of groups that admit context-free and indexed combings that first led me to the results presented in earlier sections. Further results in this direction are given in [8].

(2) The above theorem and proof remain valid if one replaces the hypothesis that the $\Gamma_{i}$ are biautomatic by the hypothesis that the groups have CF-bicombings with the comparable lengths property.

(3) The combings that we have been considering for the groups in Theorem 4.1 were obtained by reparameterizing a certain combing that had the asynchronous fellow-traveller property. Arguing as in the last paragraph of the proof of the above theorem, one sees that the image of this asynchronous combing is context free.

\section{References}

[1] A. V. Aho, Indexed grammars - an extension of context-free grammars, J. Assoc. Comp. Mach. 15 (1968), 647-671.

[2] J. Alonso and M. R. Bridson, Semihyperbolic groups, Proc. London Math. Soc. 70 (1995), 56-114.

[3] G. Baumslag, M. R. Bridson, C. F. Miller III and H. Short, Subgroups of automatic groups and their isoperimetric functions, J. London Math. Soc. 56 (1997), 292-304.

[4] G. Baumslag, M. R. Bridson, C. F. Miller III and H. Short, Fibre products, non-positive curvature and decision problems, Comment. Math. Helv. 75 (2000), 457-477.

[5] M. Bestvina and N. Brady, Morse theory and finiteness properties of groups, Invent. Math. 129 (1997), 445-470.

[6] M. R. Bridson, On the geometry of normal forms in discrete groups, Proc. London Math Soc. (3) 67 (1993), 596-616.

[7] M. R. Bridson, Regular combings, nonpositive curvature and the quasiconvexity of abelian subgroups, J. Pure and Appl. Alg. 88 (1993), 23-35.

[8] M. R. Bridson, A note on the grammar of combings, Preprint, University of Oxford, December 1999

[9] M. R. Bridson and R. Gilman, A remark about combings, Internat. J. Algebra Comput. 3 (1993), 575-581.

[10] M. R. Bridson and R. Gilman, Formal language theory and the geometry of 3-manifolds, Comment. Math. Helv. 71 (1996), 525-555.

[11] M. R. Bridson and R. Gilman, Context-free languages of sub-exponential growth, J. Comput. System Sci. 64 (2002), 308-310.

[12] M. R. Bridson and A. Haefliger, Metric Spaces of Non-Positive Curvature, Grund. Math. Wiss. 319, Springer-Verlag, Berlin-Heidelberg-New York, 1999. 
[13] J. W. Cannon, The combinatorial structure of cocompact discrete hyperbolic groups, Geom. Dedicata 16 (1984), 123-148.

[14] M. J. Dunwoody, The accessibility of finitely presented groups, Invent. Math. 85 (1985), $449-457$.

[15] D. B. A. Epstein, with J. W. Cannon, D. F. Holt, S. V. F. Levy, M. S. Paterson and W. P. Thurston, Word Processing in Groups, A. K. Peters Publishing, Natick, Mass., 1992.

[16] S. M. Gersten (ed.), Problems on automatic groups, in: Algorithms and Classification in Group Theory (G. Baumslag and C. F. Miller III, eds.), MSRI Publ. 23, Springer-Verlag, 1992, 195-224.

[17] S. M. Gersten and H. Short, Rational subgroups of biautomatic groups, Annals of Math. 134 (1991), 125-158.

[18] R. H. Gilman, Groups with a rational cross-section, in: Combinatorial Group Theory and Topology (S. M. Gersten and J. R. Stallings eds.), Princeton Univ. Press, 1987.

[19] R. I. Grigorchuk and A. Machì, An example of an indexed language of intermediate growth, Theoret. Comput. Sci. 215 (1999), 325-327.

[20] M. Gromov, Hyperbolic groups, in: Essays on Group Theory (S. M. Gersten ed.), MSRI Publ. 8, Springer-Verlag, 1987, 75-263.

[21] M. Gromov, Asymptotic invariants of infinite groups, in: Geometric Group Theory, vol. 2 (G. Niblo and M. Roller eds.), LMS Lecture Note Series, vol. 181, Cambridge Univ. Press.

[22] J. E. Hopcroft and J. D. Ullman, Introduction to Automata Theory, Languages, and Computation, Addison-Wesley, Reading, MA, 1979.

[23] L. Mosher, Central quotients of biautomatic groups, Comment. Math. Helv. 72 (1997), $16-29$.

[24] D. E. Muller and P. E. Schupp, Groups, the theory of ends and context-free languages, $J$. Computer and System Sciences 26 (1983), 295-310.

[25] W. D. Neumann and M. Shapiro, Equivalent automatic structures and their boundaries, Internat. J. Algebra Comput. 2 (1992), 443-469.

[26] G. A. Niblo (ed.), GGT 1991 problem list, Geometric Group Theory, vol. 1, (G. A. Niblo and M. Roller eds.), LMS lecture note series, vol. 181, Cambridge Univ. Press, 1993.

[27] S. Rees, Hairdressing in groups: A survey of combings and formal languages, Geometry and Topology Monographs, vol 1. (I. Rivin, C. Rourke and C. Series, eds.), 493-505.

[28] E. Rips, Subgroups of small cancellation groups, Bull. Lond. Math. Soc. 14 (1982), 45-47.

[29] M. Shapiro and M. Stein (eds.), Some problems in geometric group theory, in: Geometric Group Theory (R. Charney, M. Davis, M. Shapiro eds.), de Gruyter, Berlin-New York, $1995,183-186$.

[30] H. Short, Groups and Combings, École Normale Supérieure de Lyon, preprint \# 29 (1990).

Martin R. Bridson

Mathematics Department

Imperial College London

London SW7 2AZ

UK

e-mail: m.bridson@imperial.ac.uk

(Received: November 30, 2001)

\section{(1) To access this journal online: \\ (4) http://www.birkhauser.ch}

